ECOLOGY, BEHAVIOR AND BIONOMICS

\title{
Exigências Térmicas de Estágios Imaturos de Galleria mellonella L. (Lepidoptera: Pyralidae)
}

\author{
Aline C. Cardoso ${ }^{1}$, Márcia C. de A. Prata ${ }^{2}$, John Furlong ${ }^{2}$ e Fábio Prezoto ${ }^{1}$ \\ ${ }^{1}$ Programa de Pós-Graduação em Ciências Biológicas, Campus Universitário, Univ. Federal de Juiz de Fora \\ Martelos,36036-330, Juizde Fora, MG; ac-bio@bol.com.br,fprezoto@icb.ufjf.br \\ ${ }^{2}$ Embrapa Gado de Leite, Rua Eugênio do Nascimento, 610, Dom Bosco, 36038-330, Juiz de Fora, MG \\ mprata@cnpgl.embrapa.br,john@cnpgl.embrapa.br
}

Neotropical Entomology 36(5):657-661 (2007)

Thermal Requirements of Galleria mellonella L. (Lepidoptera: Pyralidae) Immature Stages

\begin{abstract}
The rearing of Galleria mellonella L. in laboratory is important for multiplication of entomopathogenic nematodes, mandatory for biological control studies. The objective of this study was to evaluate the effect of three thermal profiles on development stages of this insect, allowing synchronization of cycle production. Two distinct rearing phases were done: firstly, using nucleous of incubation for development of eggs and, secondly, using circular-aluminum manifolds for development of larvae and pupae. The time necessary for development of the immature stages decreased with higher temperatures. Incubation periods lasted 13.4 days at $22^{\circ} \mathrm{C}, 8.3$ at $27^{\circ} \mathrm{C}$ and 6.8 days at $32^{\circ} \mathrm{C}$, while periods for larvae development lasted 40.4, 27.2, and 23.4 days, respectively, for the same temperatures. Development to pupal stage was observed 18.2, 15.0, and 12.2 days, respectively, for the same temperatures. Larval survival was higher at $32^{\circ} \mathrm{C}$, however embryonic stages and pupae survival were higher at $27^{\circ} \mathrm{C}$. and $22^{\circ} \mathrm{C}$, respectively. The threshold temperature was $11.209167^{\circ} \mathrm{C}$ for the embryonic development stage, $7.695869^{\circ} \mathrm{C}$ for larval stage, and $1.943050^{\circ} \mathrm{C}$ for pupal stage of $G$. mellonella. Thermal constants were 138.380533 DG (degree day) for egg, 554.968830 DG for larvae, and 369.054080 DG for pupae.
\end{abstract}

KEY WORDS: Biology, temperature, entomopathogenic nematode, biological control

RESUMO - A criação de Galleria mellonella L. em laboratório é de grande importância para a multiplicação de nematóides entomopatogênicos, fundamental para estudos de controle biológico. O objetivo deste estudo foi verificar o efeito de três diferentes condições térmicas sobre os estágios imaturos desse inseto, possibilitando a sincronização de sua produção. Foram empregadas duas etapas na criação, utilizando núcleo de incubação para desenvolvimento dos ovos e recipientes circulares de alumínio para desenvolvimento de larvas e pupas. A duração dos estágios imaturos foi inversamente proporcional à temperatura em todos os bioensaios executados. Os períodos de incubação foram de 13,4 dias a $22^{\circ} \mathrm{C}, 8,3$ a $27^{\circ} \mathrm{C}$ e 6,8 a $32^{\circ} \mathrm{C}$. Para os períodos de desenvolvimento larval foram obtidos valores de 40,4, 27,2 e 23,4 dias, sob as mesmas temperaturas. O desenvolvimento pupal realizou-se em 18,2, 15,0 e 12,2 dias, na mesma ordem de temperatura. A maior sobrevivência para o estágio larval ocorreu a $32^{\circ} \mathrm{C}$; no entanto, para os estágios embrionário e pupal, as maiores sobrevivências foram registradas a $27^{\circ} \mathrm{C}$ e $22^{\circ} \mathrm{C}$, respectivamente. A temperatura base para a espécie foi de $11,209167^{\circ} \mathrm{C}$ para o desenvolvimento embrionário, $7,695869^{\circ} \mathrm{C}$ para o estágio larval e $1,943050^{\circ} \mathrm{C}$ para o pupal. As constantes térmicas foram 138,380533 GD (graus dia) para o estágio de ovo, 554,968830 GD, para larva e 369,054080 GD para pupa.

PALAVRAS-CHAVE: Temperatura, nematóide entomopatogênico, controle biológico

Conhecida como grande traça da cera, Galleria mellonella L. é um hospedeiro susceptível a nematóides entomopatogênicos dos gêneros Steinernema e Heterorhabditis, sendo por isso utilizada para multiplicação desses patógenos. Pesquisas apontam cerca de vinte espécies de nematóides com grande potencial como agentes de controle biológico de pragas agrícolas, já que eles podem localizar seus hospedeiros ativamente e infectar uma vasta gama de espécies de insetos (Dutky et al.1962, Lindegren et al. 1993).

As lagartas dessa espécie são também empregadas como hospedeiros alternativos para a criação de parasitóides 
e de outros inimigos naturais de pragas prejudiciais ao agrossistema (Gallo et al. 1988, Rijo et al. 1996). Em diversos estudos sobre fisiologia e alteração comportamental em decorrência de parasitismo, observa-se também a utilização do estádio larval de G. mellonella (Jindra \& Sehnal 1990, Wani et al. 1997).

A influência da temperatura sobre o desenvolvimento das fases imaturas dos insetos tem sido estudada para diversas espécies (Ferreira \& Parra 1985, Foerster 1996, Chagas \& Parra 2000); entretanto, são poucos os trabalhos envolvendo o ciclo biológico de G. mellonella no Brasil, utilizando-se, simultaneamente, diferentes condições térmicas.

Existe certa dificuldade na obtenção de larvas do último instar em diferentes períodos, exigindo-se, portanto, que o ciclo biológico seja sincronizado, para que tais larvas estejam disponíveis na periodicidade necessária ao desenvolvimento de experimentos com aqueles parasitas. $\mathrm{O}$ presente trabalho estudou o efeito de diferentes temperaturas sobre os estágios imaturos de G. mellonella em condições controladas, buscando possibilitar melhor manejo das criações de laboratório.

\section{Material e Métodos}

O experimento foi conduzido de outubro a dezembro de 2003, no Laboratório de Parasitologia da Embrapa Gado de Leite, Juiz de Fora, MG. Os insetos, oriundos de colônia mantida na UENF/CCTA/LPP, Campos dos Goytacazes, RJ, foram acondicionados em câmaras climatizadas tipo BOD reguladas para as temperaturas de 22,27 e $32 \pm 1^{\circ} \mathrm{C}$ e $70 \pm 10 \%$ UR, com ausência de luz. Vinte adultos machos e trinta fêmeas foram separados na câmara de acasalamento (recipiente plástico, circular; $26,6 \mathrm{~cm}$ de altura x $26 \mathrm{~cm}$ de diâmetro), no interior da qual foram dispostas folhas de papel sanfonadas, substrato adequado às fêmeas para a postura.

Estágio de ovo. Foram empregados três recipientes para incubação (três repetições) em cada temperatura testada, contendo a média de 106 ovos, dispostos na superfície de oviposição (papel) sobre um pedaço de favo. Os recipientes consistiram de pequenos potes plásticos, de $5,5 \mathrm{~cm}$ de comprimento por $5 \mathrm{~cm}$ de diâmetro, cobertos por tecido de organza e fixados com fita adesiva (para evitar eventuais fugas das larvas recém-eclodidas). Os ovos foram contados com auxílio de um microscópio estereoscópico com luz fria e um contador manual, sem manuseio direto dos mesmos.

Os ovos foram observados diariamente, pela manhã, registrando-se o dia da observação e o número de eclosões/ dia, através da contagem de ovos que externaram larvas. Tais observações permitiram a determinação do período de incubação, que compreende a média ponderada do número de dias entre a submissão dos ovos nas câmaras climatizadas e a obtenção de cada larva em cada unidade experimental. Foram analisados também o ritmo e o percentual de eclosão. O primeiro consistiu no número de eclosões/dia sobre o total de ovos submetidos, em cada unidade experimental. $\mathrm{O}$ segundo foi determinado calculando-se a porcentagem de ovos dos quais eclodiram larvas, sobre o total de ovos incubados.
Estágio larval. Cerca de dez dias após a eclosão da primeira larva, estas foram transferidas dos recipientes de incubação para recipientes de alumínio circulares (30 cm de diâmetro) contendo favo e dieta artificial (Guerra 1973) de igual proporção. Para esse estágio, avaliou-se o período larval, compreendido desde a eclosão da primeira larva até o início da formação de cada casulo, em cada unidade experimental, e a sobrevivência larval (porcentagem de larvas que originaram pupas em relação ao total de larvas obtidas, em cada unidade experimental).

Estágio pupal. As pupas foram contadas e registradas nos respectivos tratamentos. Tal procedimento possibilitou a determinação do período pupal, que compreende os dias decorridos entre o início da formação do primeiro casulo e a emergência de cada adulto, em cada unidade experimental. Além do referido período, foram analisados, para esse estágio, a sobrevivência pupal, definida como a porcentagem de adultos emergidos sobre o total de casulos considerados e representa o número de pupas viáveis para o período considerado na unidade experimental (Guerra 1973), e o ritmo de pupação, que representa o percentual de pupas formadas por dia sobre o total de larvas obtidas em cada grupo (Fraenkel \& Bhaskaran 1973).

Exigências térmicas de G. mellonella. Através da recíproca do desenvolvimento obtida por meio da equação da reta, a temperatura base $(\mathrm{Tb})$ e a constante térmica $(\mathrm{K})$ foram calculadas para os estágios imaturos de G. mellonella, utilizando-se o método de Bean (1961), utilizando-se seis casas decimais, conforme preconizado por Haddad \& Parra (1984).

Delineamento experimental. Foi utilizado o delineamento experimental inteiramente casualizado, considerando-se as três temperaturas mencionadas como tratamentos. Cada grupo de ovos (formado no máximo $24 \mathrm{~h}$ após o início da postura) correspondeu a uma unidade experimental, no total de três grupos (ou repetições) por temperatura. Critério semelhante foi empregado para larvas e pupas, totalizando três repetições por tratamento, conforme metodologia utilizada por Aguiar-Valgode \& Milward-de-Azevedo (1992) e Milward-de-Azevedo et al. (1995), priorizando-se a constituição da unidade experimental, no entanto, sem a possibilidade de realização da análise estatística dos dados.

\section{Resultados e Discussão}

Estágio de ovo. A influência da temperatura foi demonstrada em todos os bioensaios. O período médio de incubação e a viabilidade dos ovos observados nas temperaturas-teste são apresentados na Tabela 1 . O período de incubação variou de $6,8\left(32^{\circ} \mathrm{C}\right)$ a 13,4 dias $\left(22^{\circ} \mathrm{C}\right)$, sendo decrescente com a elevação térmica, na faixa testada. Esses resultados são similares aos encontrados por Abid et al. (1979), que investigaram o ciclo biológico do lepidóptero. Segundo os autores, na faixa próxima de $22^{\circ} \mathrm{C}$, o período de incubação foi, em média, 13,6 dias.

Em experimentos conduzidos por Guerra (1973) e Brar et al. (1996) a $32^{\circ} \mathrm{C}$, com a mesma espécie, foram 
Tabela 1. Tempo de desenvolvimento (em dias) dos estágios de ovo, larva e pupa de G. mellonella e suas respectivas sobrevivências (\%) ( $\mathrm{M} \pm$ desvio padrão), submetidos às temperaturas de 22,27 e $32^{\circ} \mathrm{C}$, UR $70 \pm 10 \%$; em ausência de luz.

\begin{tabular}{ccc}
\hline \multirow{2}{*}{$\begin{array}{c}\text { Temperatura } \\
\left({ }^{\circ} \mathrm{C}\right)\end{array}$} & $\begin{array}{c}\text { Desenvolvimento } \\
\text { (dias) }\end{array}$ & $\begin{array}{c}\text { Sobrevivência } \\
(\%)\end{array}$ \\
\cline { 2 - 3 } & $13,4 \pm 0,11$ & $96,8 \pm 0,38$ \\
22 & $8,3 \pm 0,10$ & $97,3 \pm 1,40$ \\
32 & $6,8 \pm 0,82$ & $78,9 \pm 32,24$ \\
\hline
\end{tabular}

\begin{tabular}{|c|c|c|}
\hline \multirow[b]{2}{*}{$\begin{array}{c}\text { Temperatura } \\
\left({ }^{\circ} \mathrm{C}\right)\end{array}$} & \multicolumn{2}{|c|}{ Larva } \\
\hline & $\begin{array}{c}\text { Desenvolvimento } \\
\text { (dias) }\end{array}$ & $\begin{array}{c}\text { Sobrevivência } \\
(\%)\end{array}$ \\
\hline 22 & $40,4 \pm 3,00$ & $78,3 \pm 20,44$ \\
\hline 27 & $27,2 \pm 1,78$ & $89,2 \pm 6,02$ \\
\hline 32 & $23,4 \pm 1,30$ & $96,6 \pm 4,11$ \\
\hline
\end{tabular}

\begin{tabular}{ccc}
\hline \multirow{2}{*}{$\begin{array}{c}\text { Temperatura } \\
\left({ }^{\circ} \mathrm{C}\right)\end{array}$} & $\begin{array}{c}\text { Pesenvolvimento } \\
\text { (dias) }\end{array}$ & $\begin{array}{c}\text { Sobrevivência } \\
(\%)\end{array}$ \\
\cline { 2 - 3 } & $18,2 \pm 2,15$ & $95,7 \pm 2,60$ \\
22 & $15,0 \pm 0,03$ & $87,4 \pm 8,64$ \\
32 & $12,2 \pm 3,14$ & $70,9 \pm 16,87$ \\
\hline
\end{tabular}

Obs. Impossibilidade de realização da análise estatística dos dados em função do pequeno número de repetições.

registradas durações médias para o estágio em questão, de 4,0 e 4,85 dias, respectivamente. Os valores observados no presente trabalho são próximos a esses. Com relação ao percentual de eclosão, Brar et al. (1996) constataram 74\% sob a temperatura de $32^{\circ} \mathrm{C}$, da qual o valor encontrado neste estudo $(70,9 \%)$ se aproximou muito. Entretanto, Warren \& Huddleston (1962), estudando a biologia de G. mellonella registraram o percentual de eclosão de $33 \%$, o que, segundo os pesquisadores, se justificou devido à baixa umidade relativa (em torno de $30 \%$ ) a que os ovos foram submetidos, provocando seu ressecamento.

Os resultados desse estudo discordam dos obtidos por Andrews (1922) que mencionou uma duração média acentuadamente maior ( 25 dias) a $24^{\circ} \mathrm{C}$ e uma viabilidade menor $(50 \%)$ a $37^{\circ} \mathrm{C}$. As razões para explicar essas diferenças dizem respeito, provavelmente, ao efeito das diferentes condições térmicas em que aquele trabalho foi conduzido e também à provável influência negativa de temperaturas mais elevadas que impediram a embriogênese.

Pelos resultados obtidos, a temperatura de $27^{\circ} \mathrm{C}$ foi a mais adequada ao desenvolvimento da fase de ovo, pela maior viabilidade (97,3\%), o que representa maior número de ovos, assegurando que seja recomendada para a manutenção de colônias de G. mellonella. O menor percentual de eclosão observado ocorreu a $32^{\circ} \mathrm{C}(78,9 \%$, em média), embora, nessa temperatura, o período de incubação tenha sido menor, o que minimiza o tempo de desenvolvimento na criação.

O ritmo de eclosão das larvas de $G$. mellonella encontrase na Fig. 1. Seis dias após o início do experimento, 65,9\% das larvas, a $32^{\circ} \mathrm{C}$, já haviam eclodido. Esses dados se aproximam dos obtidos por Guerra (1973), que verificou o maior índice de eclosão, a $32^{\circ} \mathrm{C}$, no período de $96 \mathrm{~h}$ a $120 \mathrm{~h}$ (cinco a seis dias). A maior taxa de eclosão, a $27^{\circ} \mathrm{C}$, ocorreu no oitavo dia (51,2\%); no entanto, o pico de eclosão (83\%), a $22^{\circ} \mathrm{C}$, ocorreu em torno de 13 dias após o início da pesquisa, corroborando estudos anteriores que relacionam temperaturas baixas à desaceleração na taxa de metabolismo, resultando em períodos mais longos para os diferentes parâmetros biológicos (Aguiar-Valgode \& Milward-de-Azevedo 1992, Milward-de-Azevedo et al. 1995).

Estágio larval. A duração do estágio larval foi decrescente na faixa de temperatura avaliada (Tabela 1), com durações médias variáveis de $40,4\left(22^{\circ} \mathrm{C}\right)$ a 23,4 dias $\left(32^{\circ} \mathrm{C}\right)$. Esses valores foram, em geral, menores do que os relatados por El-Sawaf (1950) e Mishra (1971). O primeiro encontrou a média de 68,37 dias a $29,6^{\circ} \mathrm{C}$ e um aumento para 225 dias quando a temperatura decresceu para $18,6^{\circ} \mathrm{C}$. Os registros do segundo autor foram de 105 dias a $20^{\circ} \mathrm{C}, 39,5$ a $26,1^{\circ} \mathrm{C}$ e 39 dias a $31,8^{\circ} \mathrm{C}$.

Os resultados concordam com os limites determinados por Flechtmann (1964) e Guerra (1973). O primeiro autor registrou 24,4 dias para o desenvolvimento de larvas criadas à temperatura de $27,6^{\circ} \mathrm{C}$. O autor registrou duração média de 30,5 dias a $32^{\circ} \mathrm{C}$, utilizando condições similares às previstas no presente estudo.

A maior sobrevivência foi obtida a $32^{\circ} \mathrm{C}(96,7 \%$; Tabela 1$)$, indicando ser esta temperatura ideal para o desenvolvimento de larvas da mariposa da cera, por apresentar também a menor duração dessa fase, viabilizando sua criação em laboratório Esse valor encontrou-se muito acima do relatado por Brar et al. (1996) que constataram uma viabilidade larval variando de $60 \%$ a $65 \%$, na mesma condição térmica. Assim, podese utilizar a temperatura de $22^{\circ} \mathrm{C}$ quando se desejar retardar

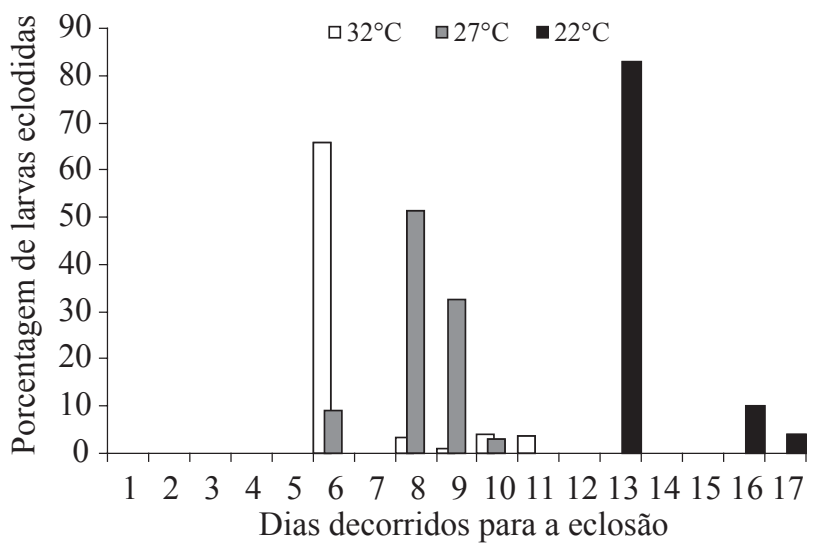

Fig. 1. Ritmo de eclosão de larvas de G. mellonella, a 22, 27 e $32^{\circ} \mathrm{C}$. UR $70 \pm 10 \%$; em ausência de luz. 
a velocidade de desenvolvimento, aumentando o tempo de utilização das larvas em laboratório, sem prejuízo ao desempenho biológico do inseto.

Estágio pupal. Foram registradas durações médias de 18,2 dias a $22^{\circ} \mathrm{C}, 15,0$ dias a $27^{\circ} \mathrm{C}$ e 12,2 dias a $32^{\circ} \mathrm{C}$ (Tabela 1 ). Esses dados discordam dos obtidos por Brar et al. (1996), que encontraram valores menores para o período ( 4,5 a 4,8 dias), a $32^{\circ} \mathrm{C}$, porém a sobrevivência pupal foi semelhante $(65 \%)$ No entanto, a $32^{\circ} \mathrm{C}$, a sobrevivência obtida neste trabalho $(70,9 \%)$ foi superior à relatada por Guerra (1973), que foi de $44,85 \%$, na mesma temperatura.

O ritmo de pupação é apresentado na Fig. 2. Os maiores índices foram observados sete dias (44,3\%) e oito dias (35\%) após a formação da primeira pupa em espécimes submetidos a $32^{\circ} \mathrm{C}$ e $27^{\circ} \mathrm{C}$, respectivamente. No terceiro dia após a identificação da primeira pupa foi registrada a maior taxa, a $22^{\circ} \mathrm{C}(22,6 \%)$. A ausência de estudos anteriores com $G$. mellonella referentes a esse parâmetro, inviabilizou possíveis comparações dos resultados.

Â semelhança da fase larval, a melhor condição térmica para o desenvolvimento de pupas, foi $32^{\circ} \mathrm{C}$, embora a sobrevivência tenha sido menor do que nas outras temperaturas. $\mathrm{O}$ estágio de pupa apresentou a maior sobrevivência a $22^{\circ} \mathrm{C}$, garantindo o maior número de adultos para o manejo das colônias em laboratório.

A Fig. 3 contém o resumo dos períodos médios relacionados aos estágios imaturos de G. mellonella, nas três condições térmicas utilizadas neste trabalho.

Exigências térmicas de $\boldsymbol{G}$. mellonella. Os parâmetros relativos às exigências térmicas dos estágios imaturos de $G$. mellonella encontram-se na Tabela 2. O limite inferior de temperatura $(\mathrm{Tb})$ para a espécie foi de $7,695869^{\circ} \mathrm{C}$ no estágio larval, indicando que larvas submetidas a temperaturas abaixo desse limiar, cessam completamente suas atividades. Para os estágios de ovo e pupa foram registrados, para a temperatura base, 11,209167 e $1,943050^{\circ} \mathrm{C}$, respectivamente.

Através do valor obtido para a constante térmica relativa ao estágio larval (554,968830 GD) e da temperatura base, é possível estimar-se a duração do estágio, aplicando-se a equação $1 / \mathrm{D}=-0,013867+0,001802 . \mathrm{T}$, onde "T" é a temperatura média, em graus Celsius, do período a ser avaliado e "D" é a duração, em dias, do estágio larval na temperatura "T". Do mesmo modo, a aplicação das equações definidas para os estágios de ovo e pupa, é válida para se estimar a duração dos mesmos. Considera-se o reconhecimento das exigências térmicas dos hospedeiros como subsídio para o manejo integrado de pragas. Embora neste estudo tenham sido empregadas apenas três temperaturas para a determinação das exigências térmicas de G. mellonella, pelo método de Bean (1961), através do cálculo do coeficiente de correlação (r), demonstrou-se a viabilidade dessa técnica, uma vez que os valores de $\mathrm{r}$ foram próximos de 1, confirmando sua confiabilidade.

Considerando os resultados acima e os parâmetros "velocidade de desenvolvimento" e "produtividade" na obtenção dos estágios de G. mellonella, propõe-se a

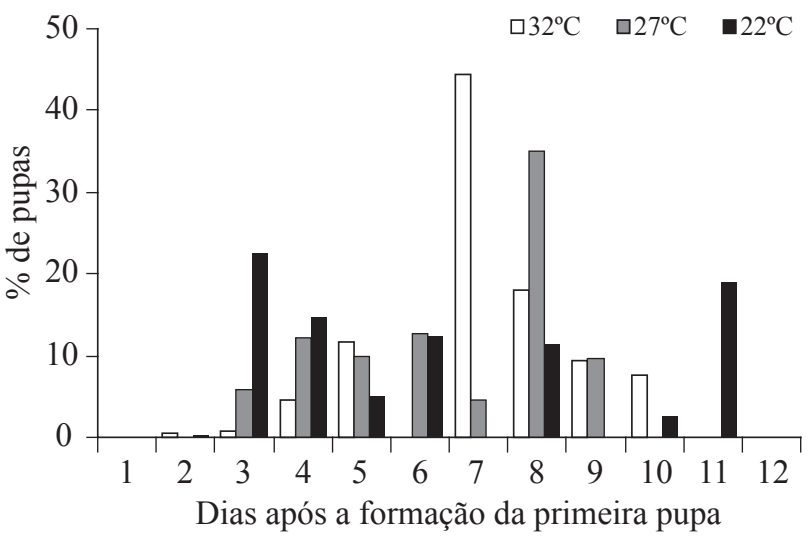

Fig. 2. Ritmo de pupação de G. mellonella em três temperaturas $\left(22,27\right.$ e $\left.32^{\circ} \mathrm{C}\right)$. UR $70 \pm 10 \%$; em ausência de luz.

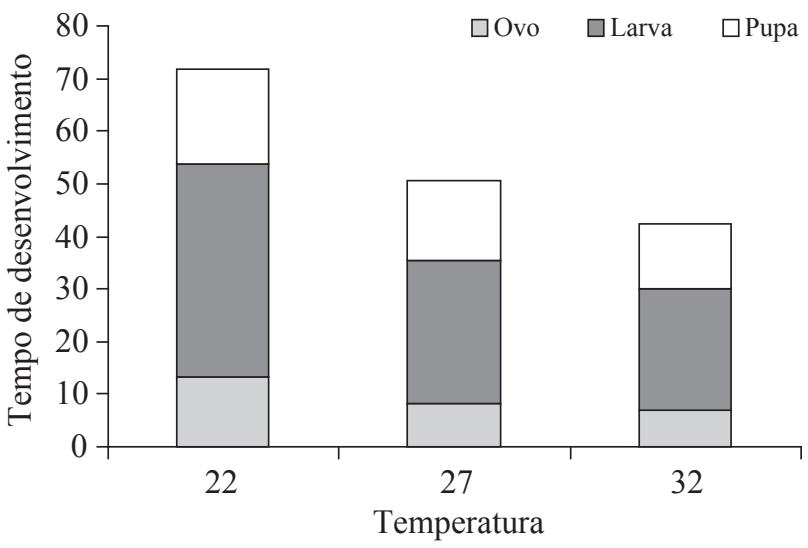

Fig. 3. Durações médias da incubação, período larval e pupal de $G$. mellonella em três temperaturas $\left(22,27\right.$ e $\left.32^{\circ} \mathrm{C}\right)$. UR 70 $\pm 10 \%$; em ausência de luz.

Tabela 2. Parâmetros relativos às exigências térmicas dos estágios de ovo, larva e pupa de G. mellonella, em laboratório, sob as temperaturas de 22,27 e $32^{\circ} \mathrm{C}$ e UR $70 \pm 10 \%$, em ausência de luz, calculados segundo método de Bean (1961).

\begin{tabular}{lccc}
\hline Parâmetro & Ovo & Larva & Pupa \\
\hline Coeficiente de correlação $(\mathrm{r})$ & 0,99 & 0,98 & 0,99 \\
Temperatura base $\mathrm{Tb}\left({ }^{\circ} \mathrm{C}\right)$ & 11,209167 & 7,695869 & 1,943050 \\
Constante térmica K (GD) & 138,380533 & 554,968830 & 369,054080 \\
Equação da reta & $1 / \mathrm{D}=-0,081002+0,007226 \mathrm{~T}$ & $1 / \mathrm{D}=-0,013870+0,001802 \mathrm{~T}$ & $1 / \mathrm{D}=-0,005265+0,002709 \mathrm{~T}$
\end{tabular}

T: temperatura $\left({ }^{\circ} \mathrm{C}\right)$; D: duração do estágio de desenvolvimento embrionário na temperatura $\mathrm{T}$ ” 
manutenção da fase de ovo a $27^{\circ} \mathrm{C}$, com maximização tanto da velocidade de desenvolvimento quanto da produtividade. Em relação à larva, e pelos mesmos motivos, a temperatura ideal é a de $32^{\circ} \mathrm{C}$. O estágio pupal, entretanto, pode ser mantido à temperatura de $22^{\circ} \mathrm{C}$ quando se prioriza o aspecto produtividade de espécimes, que foi de $95,7 \%$ comparado com $71 \%$ à temperatura de $32^{\circ} \mathrm{C}$, ou a $32^{\circ} \mathrm{C}$ quando o objetivo é velocidade de desenvolvimento, 12,2 dias comparado a 18,2 dias à temperatura de $22^{\circ} \mathrm{C}$.

\section{Referências}

Abid, M.K., M.F. Shaarawy \& M.K. El-Yassery. 1979. Studies on the biology of the greater wax moth Galleria mellonella (L.), (Lepidoptera: Galleridae). Iraq. Bull. Nat. Hist. Res. Centre 7: $1-10$.

Aguiar-Valgode, M. \& E.M.V. Milward-de-Azevedo. 1992. Determination of thermal requirements of Stomoxys calcitrans (L.) (Diptera, Muscidae), under laboratory conditions. Mem. Inst. Oswaldo Cruz 87: 11-20.

Andrews, J.E. 1922. Some experiments with the larva of the beemoth, Galleria mellonella L. Trans. Wisc. Acad. Sci. Arts. Lett. 20: 255-261.

Bean, J.L. 1961. Predicting emergence of second-instar spruce budworm larvae from hibernation under field conditions in Minnesota. Ann. Entomol. Soc. Am. 54: 175-177.

Brar, H.S., B.S. Brar, G.S. Gatoria \& H.S. Jhajj. 1996. Biology of greater wax moth, Galleria mellonella L. infesting Apis mellifera L. colonies in Punjab. J. Insect Sci. 9: 12-14.

Chagas, M.C.M. \& J.R.P. Parra. 2000. Phyllocnistis citrella Stainton (Lepidoptera: Gracillariidae): Técnica de criação e biologia em diferentes temperaturas. An. Soc. Entomol. Brasil 29: 227-235.

Dutky, S.R., J.V. Thompson \& G.E. Cantwell. 1962. A technique for mass rearing the greater wax moth (Lepidoptera: Galleriidae). Proc. Entomol. Soc. Wash. 64: 56-58.

El-Sawaf, S.K. 1950. The life-history of the greater wax-moth (Galleria mellonella L.) in Egypt, with special reference to the morphology of the mature larva. Bull. Soc. Fouad. ler Entomol. 34: 247-297.

Ferreira, M.J.M. \& J.R.P. Parra. 1985. Biologia de Mocis latipes (Guenée, 1852) (Lepidoptera: Noctuidae) em diferentes temperaturas para determinação das exigências térmicas. An. Soc. Entomol. Brasil 14: 75-88.

Foerster, L.A. 1996. Efeito da temperatura no desenvolvimento das fases imaturas de Pseudaletia sequax Franclemont (Lepidoptera: Noctuidae). An. Soc. Entomol. Brasil 25: 27-32.
Fraenkel, G. \& G. Bhaskaran. 1973. Pupariation and pupation in cyclorrhaphous flies (Diptera): Terminology and interpretation. Ann. Entomol. Soc. Am. 66: 418 - 422.

Gallo, D., O. Nakano, S. Silveira Neto, R.P.L. Carvalho, G.C. Batista, E. Berti Filho, J.R.P. Parra, R.A. Zucchi, S.B. Alves \& J.D. Vendramin. 1988. Manual de entomologia agrícola. $2^{\mathrm{a}}$. edição. Editora Agronômica Ceres Ltda., São Paulo, 649p.

Guerra, M.S. 1973. Bionomia das traças da cera Galleria mellonella L. e Achroia grisella F. (Lepidoptera - Galleriidae) no Município de Piracicaba, São Paulo. Dissertação de mestrado, ESALQ, Piracicaba, 133p.

Haddad, M.L. \& J.R.P. Parra. 1984. Métodos para estimar exigências térmicas e os limites de desenvolvimento dos insetos. Piracicaba, FEALQ, 45p.

Jindra, M. \& F. Sehnal. 1990. Linkage between diet humidity, metabolic water production and heat dissipation in the larvae of Galleria mellonella. Insect Biochem. 20: 389-395.

Lindegren, J.E., K.A. Valero \& B.E. Mackey. 1993. Simple "in vivo" production and storage methods for Steinernema carpocapsae infective juveniles. J. Nematol. 25: 193-197.

Milward-de-Azevedo, E.M.V., J. Herzog, M.A.S. Freitas \& E.H.S. Faria. 1995. Desenvolvimento ontogenético, potencial reprodutivo e longevidade de Chrysomya megacephala (Fabricius) (Diptera, Calliphoridae), em condições de laboratório. Revta Bras. Ent. 39: 623-632.

Mishra, S.C. 1971. Observations on the biology of wax moth Galleria mellonella L. (Pyralidae: Lepidoptera). Indian For. 97: 573-576.

Rijo, E., N. Matos \& A. Barrios. 1996. Desarrollo de Galleria mellonella alimentada con dieta a base de derivados de la industria azucarera. Cienc. Tec. Agric. Protec Plantas 12 : 61-69.

Wani, M., K. Iwabuchi, N. Agui \& J. Mitsuhashi. 1997. Endocrine alteration and precocious premetamorphic behaviors in the greater wax moth larvae, Galleria mellonella, parasitized by an endoparasitoid, Apanteles galleriae. Arch. Insect Biochem. Physiol. 34: 257-273.

Warren, L.O. \& P. Huddleston. 1962. Life history of the greater wax moth, Galleria mellonella L., in Arkansas. J. Kans. Entomol. Soc. 35: 212-216.

Received 15/II/06. Accepted 09/XII/06. 